\title{
Self-Assembly of Viral Capsid Proteins Driven by Compressible Nanobubbles
}

\author{
Minmin Zhang, ${ }^{*}, \#$ Shuqin Cao," Aijie Liu, Jeroen J. L. M. Cornelissen, and Serge G. Lemay* \\ Cite This: J. Phys. Chem. Lett. 2020, 11, 10421-10424 \\ Read Online
}

ABSTRACT: Colloidal nanobubbles occur in gas-saturated aqueous solutions following high power water electrolysis. Here the influence of nanobubble solutions on the self-assembly properties of viral capsid proteins (CP) was investigated. Interestingly, we found that gas solutions were able to trigger the self-assembly of CP of cowpea chlorotic mottle virus (CCMV) in the absence of the viral genome, most likely by acting as a negatively charged template. The process was demonstrated by three distinct techniques, namely, dynamic light scattering (DLS), atomic force microscopy (AFM), and transmission electron microscopy (TEM). Furthermore, nanobubble-induced self-assembly of viral $\mathrm{CP}$ was found to depend on protein concentration. Low CP concentrations led to assembly of $18 \mathrm{~nm}$ virus-like particles (VLPs), comparable to $T=1$ (Casper and Klug triangulation number) virus capsids, whereas high CP concentrations led to $28 \mathrm{~nm}$ VLPs (similar to $T=3$ capsids). This paves a new route for self-assembly of VLPs.

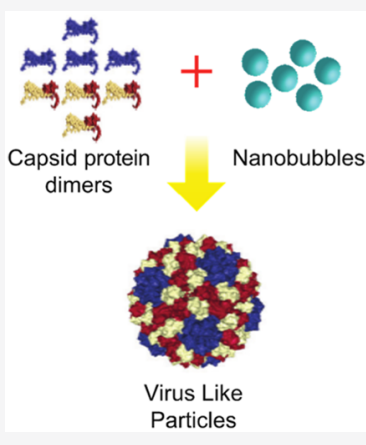

mall gas bodies with properties consistent with previously $\checkmark$ reported nanobubble findings have been observed in gassaturated solutions following high power water electrolysis. ${ }^{1-3}$ Herein we likewise refer to them as bulk nanobubbles. Bulk nanobubbles exhibit several properties that differ from macroscopic bubbles, such as lower buoyancy, which can be neglected compared with thermal energy. ${ }^{4}$ Small bubbles also exhibit large specific interfacial area, by virtue of which one can expect strong interactions of bubbles with other similarly-sized objects present in solution. In addition, bulk nanobubbles bear a negative surface charge that inhibits the agglomeration or coalescence of bubbles. In particular, we recently reported that gas bubbles interact with both negatively and positively charged nanoparticles via nucleation on nanoparticle surfaces, ${ }^{3,5}$ even causing reentrant condensation of positive particles upon mixing with gas-saturated solutions. ${ }^{5}$

Self-assembled viral protein cages have attracted great interest because of their controlled assembly and disassembly properties as well as their potential as biomolecular scaffolds with a highly organized structure. ${ }^{6}$ They are widely used as drug carriers and nanoreactors. ${ }^{7-10}$ CCMV is a member of the Bromoviridae family, a plant virus with an icosahedral shell of $28 \mathrm{~nm}$ outer diameter and $18 \mathrm{~nm}$ inner diameter that surrounds a central core of single-stranded viral RNA. ${ }^{11} \mathrm{~A}$ particular interesting characteristic of CCMV is that native CCMV can disassemble into CPs and its genome RNA in vitro. ${ }^{12}$ After removal of RNA, CPs can be reassembled into virus-like particles (VLPs) either by reducing $\mathrm{pH}$ or by introducing negatively charged inorganic nanoparticles and synthetic anionic polymers. The structure and size of VLPs varies from tubes to icosahedral capsids arranged with Caspar and Klug triangulation number $T=1(60 \mathrm{CP}$ dimers, $18 \mathrm{~nm})$, $T=2(120$ dimers, $22 \mathrm{~nm})$, and $T=3(180$ dimers, $28 \mathrm{~nm})$ symmetry. ${ }^{6,12-14}$ In this respect, CCMV offers the versatility to realize nanoassemblies of different sizes and nanoscale structure.

Inorganic nanoparticles of various types and functions have been widely used as templates to grow VLPs. ${ }^{6,7,15}$ However, a metastable soft template such as a nanobubble has never been used to guide the growth of VLPs. A primary goal of this study was to test whether nanobubbles, which exhibit a negative surface charge, can also be used as a template to trigger the formation of VLPs.

Characterization of Self-Assembled VLPs Driven by Compressible Nanobubbles. Herein we demonstrate the controlled assembly of cowpea chlorotic mottle virus (CCMV)-based viral capsid proteins using bulk nanobubbles as template. The experimental route for nanobubble-induced assembly of VLPs is presented in Figure 1. The virus dissociated into RNA and protein subunits when it was dialyzed against $0.5 \mathrm{M} \mathrm{CaCl}_{2}$ at $\mathrm{pH}$ 7.5. ${ }^{16}$ Isolated capsid protein dimers were purified and stored in Tris buffer. Nanobubble-triggered self-assembly of VLPs was achieved by adding $400 \mu \mathrm{L}$ freshly generated nanobubble solutions at a concentration of $10^{8} / \mathrm{mL}$ into 200 $\mu \mathrm{L}$ of capsid proteins $(1-5 \mathrm{mg} / \mathrm{mL})(2: 1$ bubble-protein volume ratio) and incubated overnight.

We first examined the assembly of CPs in the presence of a negatively charged nanobubble solution (zeta potential $\zeta=$

Received: August 31, 2020

Accepted: November 9, 2020

Published: December 3, 2020 


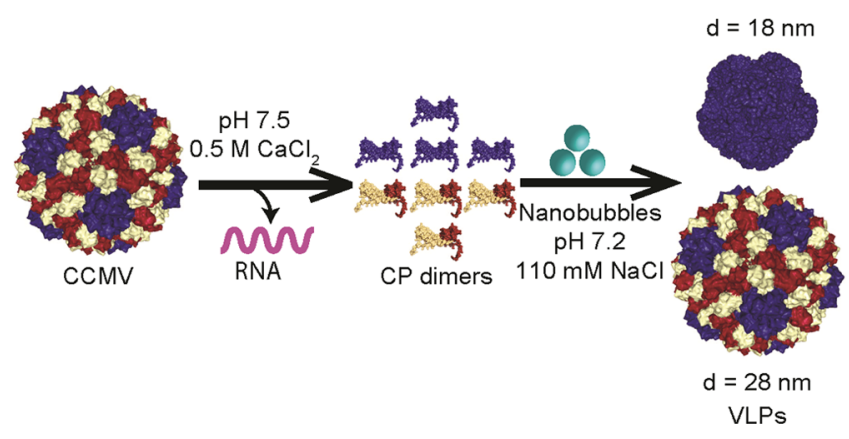

Figure 1. Schematic representation of nanobubble-triggered selfassembly of virus-like particles (VLPs) from viral capsid proteins (CPs). Low CP concentration leads to assembly of $18 \mathrm{~nm}$ VLPs, whereas high CP concentration leads to $28 \mathrm{~nm}$ VLPs.

$-23 \pm 7 \mathrm{mV}$ ) with dynamic light scattering (DLS). We hypothesized that nanobubbles with a size around $200 \mathrm{~nm}$ (Figure 2a) were most likely to yield similarly sized VLPs, as widely reported using incompressible templates. ${ }^{6,10,15}$ Instead, interestingly, the nanobubble solution triggered self-assembly of CPs into VLPs with a diameter of $26 \mathrm{~nm}$, which is similar to the native CCMV virus (Figure $2 b, c$ ). In contrast, in the control experiment, mixing with a non-electrolysis treated 10 $\mathrm{mM} \mathrm{NaCl}$ solution yielded no VLPs (Figure 2d), indicating that indeed the self-assembly of CPs into VLPs at neutral $\mathrm{pH}$ (7.2) was driven by nanobubbles.

Both AFM and TEM imaging were also used to verify the formation of VLPs (Figure 3). As depicted in Figure 3a, a considerable number of VLP assemblies could be imaged by AFM. The histogram of the measured height of the particles (Figure 3b) revealed a mean diameter of $27 \mathrm{~nm}$, which matches well with the hydrodynamic VLP diameter from DLS. The same sample was also analyzed by TEM. Similar to both DLS and AFM results, we observed assembly of VLPs with a mean diameter of $30 \mathrm{~nm}$ (Figure 3d). Given the identical results from three distinct techniques, this strongly suggests that self-assembly of CPs into VLPs is induced by nanobubbles.
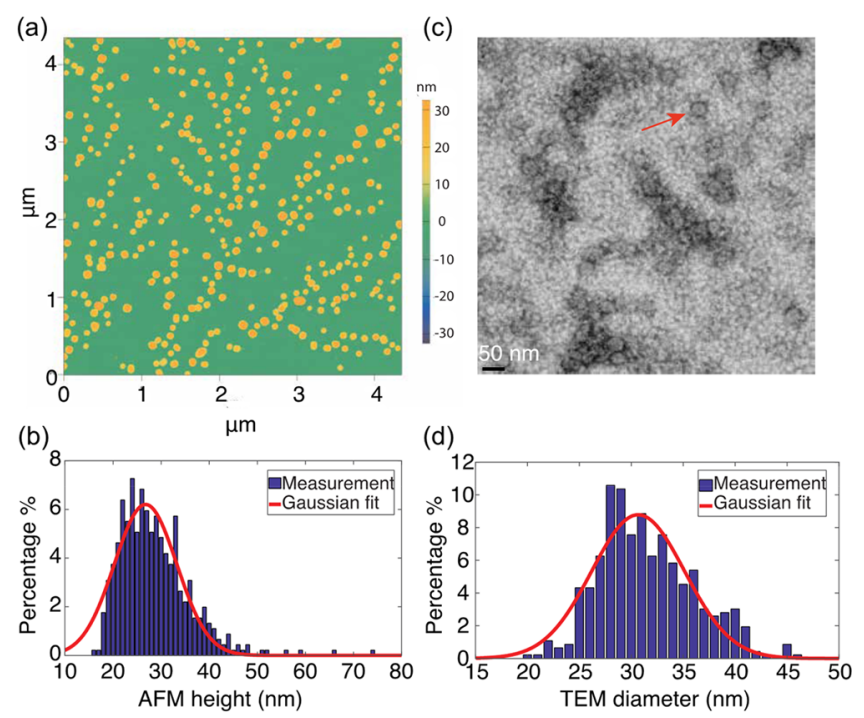

Figure 3. Analytical data of nanobubble induced self-assembly of virus like particles. (a) Atomic force microscopy image of VLPs and (b) their size distributions based on 454 counts. (c) Negatively stained transmission electron microscopy image of VLPs and (d) their size distributions based on 463 counts.

Self-Assembly Is Controlled by the Amount of Gas. Since VLP formation is induced by the nanobubble solution, it was anticipated that the removal of gas from the solution should be accompanied by a drop in efficiency or even failure of selfassembly of VLPs. To demonstrate this, we investigated whether CPs lacking negatively charged nanobubbles could self-assemble into VLPs under the same conditions. As shown in Figure S1a, when the nanobubble solution was degassed by a vacuum pump for $3 \mathrm{~h}$, the peak in the size distribution at 26 $\mathrm{nm}$ exhibited relatively low intensity and coexisted with another size peak located at $1 \mathrm{~nm}$. When the nanobubble solution was degassed for a longer period $(5 \mathrm{~h})$, only a single size distribution at $1 \mathrm{~nm}$ corresponding to pure $\mathrm{CP}$ solution was observed (Figure S1b). This is highly consistent with the
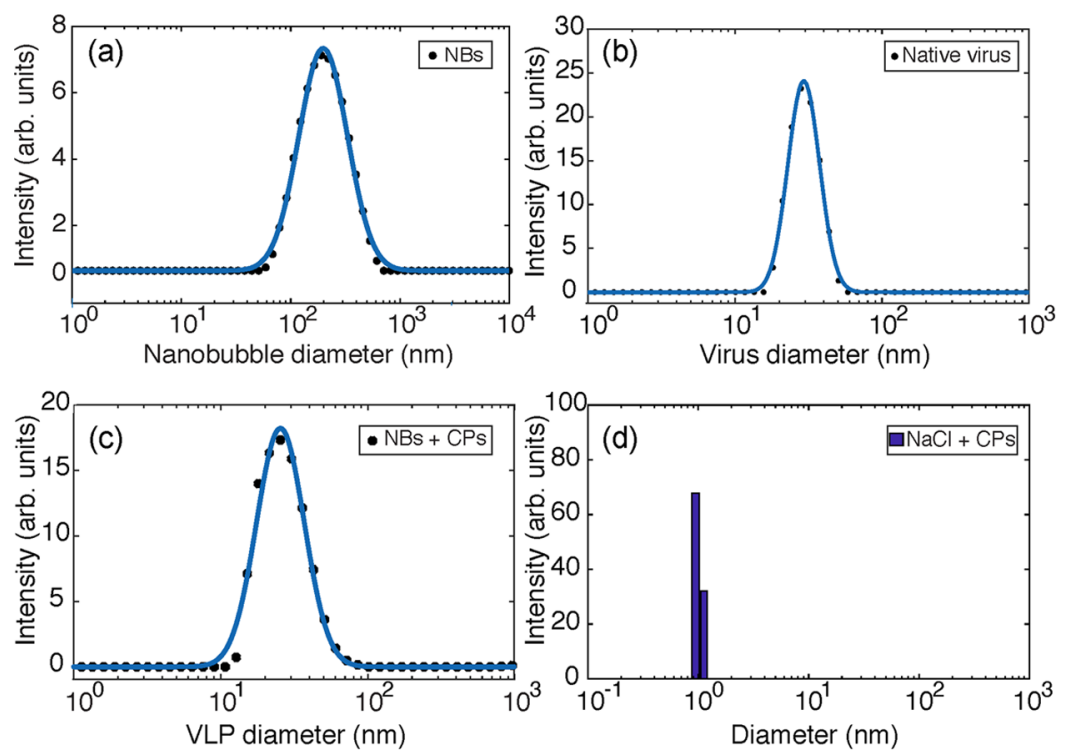

Figure 2. Hydrodynamic diameter of (a) pure nanobubbles, (b) native CCMV virus, (c) self-assembled VLPs induced by nanobubbles (NBs), and (d) blank in which CPs mixed with an untreated $10 \mathrm{mM} \mathrm{NaCl}$ solution. All other parameters were kept the same throughout. 
DLS results for the control with a $\mathrm{CP}-\mathrm{NaCl}$ mixture (Figure 2d). Combining those two observations clearly demonstrates that removal of gas from bubble solution leads to failure of the assembly of VLPs and thus that gas oversaturation plays an important role in VLP self-assembly process.

The structure and stability of the polymorphs of CCMVbased CP assembly can be comprehended in terms of electrostatic interactions and the way they affect the spontaneous curvature of protein networks. ${ }^{17-19}$ There are two types of interactions within the protein particle: the interaction between neighboring $\mathrm{CPs}(\mathrm{CP}-\mathrm{CP})$ and the interaction between $\mathrm{CP}$ and cargo $(\mathrm{CP}-$ cargo). The entire assembly process depends on the strength of $\mathrm{CP}-\mathrm{CP}$ attraction relative to $\mathrm{CP}-$ cargo attraction. $\mathrm{CP}-\mathrm{CP}$ interactions come from a combination of hydrophobic attraction and electrostatic repulsion. $\mathrm{CP}$-cargo interactions are more sensitive to ionic strength. The basic residues at the $\mathrm{N}$ terminus of $\mathrm{CP}$ have high $\mathrm{p} K_{\mathrm{a}}$ values so that they remain positively charged throughout the $\mathrm{pH}$ range that we studied. Further studies and analysis on electrostatic attractions between positively charged N-terminus of $\mathrm{CP}$ and negatively charged cargo were pursued as follows.

Self-Assembly of VLPs depends on CP concentration. To study $\mathrm{CP}-\mathrm{NB}$ interactions, we explored the role of $\mathrm{CP}$ concentration in the assembly. We characterized the assembly of VLPs with different $\mathrm{CP}$ concentrations while keeping the $\mathrm{pH}$ and ionic strength of the mixture $(\mathrm{pH} 7.2,110 \mathrm{mM} \mathrm{NaCl})$ and the $\zeta$ potential of the nanobubbles $(-20 \mathrm{mV})$ constant. When $1 \mathrm{mg} /$ $\mathrm{mL} C P$ was mixed with nanobubble solution with a concentration of $10^{8} / \mathrm{mL}$ at regular volume ratio, the NBCP formed VLPs with a peak diameter of $18 \mathrm{~nm}$ (Figure 4 red

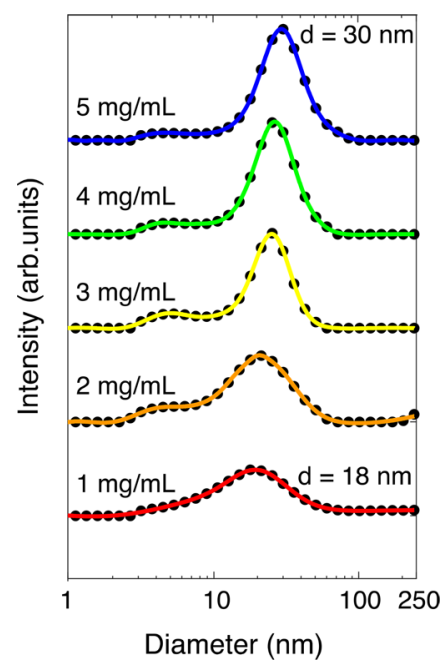

Figure 4. Size distribution of self-assembled VLPs as a function of CP concentration. At $1 \mathrm{mg} / \mathrm{mL}$, VLPs of $18 \mathrm{~nm}$ diameter (similar to $T=$ 1) were assembled, whereas VLPs of $30 \mathrm{~nm}$ diameter (similar to $T=$ 3) were assembled with $\mathrm{CP}$ at $5 \mathrm{mg} / \mathrm{mL}$. The crossover occurred for $\mathrm{CP}$ concentration between 1 and $5 \mathrm{mg} / \mathrm{mL}$.

curve), suggesting a structure similar to $T=1$ (60 CP dimers, $18 \mathrm{~nm})$. Increasing the concentration of $\mathrm{CP}$ to $2 \mathrm{mg} / \mathrm{mL}$, we observed assembly of VLPs with a peak diameter of $21 \mathrm{~nm}$ (Figure 4 orange curve), which is similar to the diameter of the $T=2$ structure (120 dimers, $22 \mathrm{~nm}$ ). Further increasing CP concentration up to 3,4 , and $5 \mathrm{mg} / \mathrm{mL}$, peak diameters of 26 , 27 , and $30 \mathrm{~nm}$ were obtained, respectively (Figure 4 yellow, green and blue curve, respectively), similar to the $T=3$ structure (180 dimers, $28 \mathrm{~nm}$ ). Once CP is higher than $5 \mathrm{mg} /$ $\mathrm{mL}$, the peak diameter of VLPs remained essentially constant around $30 \mathrm{~nm}$, no further significant increase in size was observed. These results suggest that the size distribution of VLPs reflects the assembly mechanism with the optimal size being dictated by the concentration of CP. No self-assembly products were obtained with CP concentrations below $1 \mathrm{mg} /$ $\mathrm{mL}$, presumably due to the low CP supply.

Self-Assembly of VLPs Is Charge Dependent and Reversible. To further verify that $\mathrm{CP}-\mathrm{NB}$ interaction is the main reason for self-assembly of VLPs, we carried out a competition study to examine whether the amount of charge of NB affects the assembly as shown in Figure S2. We were able to tune the $\zeta$ potential of nanobubbles in solution via changing the generation parameters, especially the supersaturation level after generation. We found that for NBs at a $\zeta$ potential of -13 $\mathrm{mV}$, most of CPs remain disassembled. For NBs with a $\zeta$ potential of $-20 \mathrm{mV}$; on the other hand, most of the CPs selfassembled into VLPs with a diameter of $30 \mathrm{~nm}$, again suggesting an architecture similar to $T=3$. The inability of CPs to assemble at $\zeta$ potential of $-13 \mathrm{mV}$ indicates that the lack of charge matching between the $\mathrm{N}$-terminus of CPs and the negatively charged NBs lowers the attractive forces of CP$\mathrm{NB}$, thus lowering the VLP formation efficiency. This is reminiscent of the encapsulation of genomic material, as a universal charge ratio of 1.6 for genome to total capsid is needed for most RNA viruses. ${ }^{21,22}$

Another observation supporting $\mathrm{CP}-\mathrm{NB}$ interaction is that assembly of VLPs is reversible (Figure S3). Figure S3a shows that after a week of VLP assembly, the capsid solution started to become unstable and a size distribution below $10 \mathrm{~nm}$ corresponding to hexameric or pentameric subunits appeared. However, upon adding an extra amount of negatively charged nanobubble solution $(400 \mu \mathrm{L})$ to the unstable capsid solution, the disassembled CP reassembled back into intact VLPs (Figure S3b). This observation demonstrates the assembly is reversible when the NB solution becomes less saturated, strongly suggesting that gas remains present in the NBstabilized VLPs.

However, electrostatic interactions are not the only important contributor to VLP assembly in the CP-NB system. While we cannot yet describe the exact pathway for $\mathrm{CP}-\mathrm{NB}$ assembly into the Kaspar Clug symmetry, ${ }^{20}$ the observations suggest that NBs facilitate the nucleation of VLPs. This could occur via the growth of a single proto-VLP or by driving the formation of hexameric and pentameric subunits which then combine.

In conclusion, we introduced a simple route for the formation of CCMV-based VLPs driven by compressible nanobubbles via $\mathrm{CP}-\mathrm{NB}$ attractions at neutral $\mathrm{pH}$ and low ionic strength. Three distinct characterization techniques were employed to demonstrate the successful assembly of VLPs. The assembly was found to depend on CP concentration. Furthermore, the assembly of VLPs could be attenuated by lower NB surface charges and was reversible upon decrease in gas supersaturation. Controlling the assembly of a virus capsid with compressible nanobubbles enables the formation of welldefined protein architecture induced by a distinct new template, whose size and geometry does not need to correspond to the shape and size of the final product. 


\section{ASSOCIATED CONTENT}

\section{s) Supporting Information}

The Supporting Information is available free of charge at https://pubs.acs.org/doi/10.1021/acs.jpclett.0c02658.

Generation and characterization of both CCMV capsid protein and nanobubble solutions; self-assembly and characterization of VLPs; the influence of amount of gas on DLS, NB surface charge and supersaturation of NB solution on VLP assembly (PDF)

\section{AUTHOR INFORMATION}

\section{Corresponding Authors}

Minmin Zhang - Guangdong Provincial Key Laboratory of Nanophotonic Functional Materials and Devices, School of Information and Optoelectronic Science and Engineering, South China Normal University, Guangzhou 510006, China; Bioelectronics Research Group, MESA+ Institute for Nanotechnology, University of Twente, Enschede 7500, AE, The Netherlands; 이잉.org/0000-0002-2211-2850; Email: zhangminmin@m.scnu.edu.cn

Serge G. Lemay - Bioelectronics Research Group, MESA+ Institute for Nanotechnology, University of Twente, Enschede 7500, AE, The Netherlands; (1) orcid.org/0000-0002-04043169; Email: s.g.lemay@utwente.nl

\section{Authors}

Shuqin Cao - Laboratory for Biomolecular Nanotechnology, MESA+ Institute for Nanotechnology, University of Twente, Enschede 7500, AE, The Netherlands

Aijie Liu - Laboratory for Biomolecular Nanotechnology, MESA+ Institute for Nanotechnology, University of Twente, Enschede 7500, AE, The Netherlands; (1) orcid.org/00000002-0300-1872

Jeroen J. L. M. Cornelissen - Laboratory for Biomolecular Nanotechnology, MESA+ Institute for Nanotechnology, University of Twente, Enschede 7500, AE, The Netherlands; (ㄱ) orcid.org/0000-0002-9728-5043

Complete contact information is available at:

https://pubs.acs.org/10.1021/acs.jpclett.0c02658

\section{Author Contributions \\ ${ }^{\#}$ M.Z and S.C. contributed equally. \\ Notes}

The authors declare no competing financial interest.

\section{ACKNOWLEDGMENTS}

We thank the MESA+ Institute for Nanotechnology of the University of Twente and the Tennant Company for financial support.

\section{REFERENCES}

(1) Postnikov, A. V.; Uvarov, I. V.; Lokhanin, M. V.; Svetovoy, V. B. Electrically controlled cloud of bulk nanobubbles in water solutions. PLoS One 2017, 12, e0181727.

(2) Alheshibri, M.; Qian, J.; Jehannin, M.; Craig, V. S. J. A history of nanobubbles. Langmuir 2016, 32, 11086-11100.

(3) Zhang, M.; Seddon, J. R. T. Nanobubble-Nanoparticle Interactions in Bulk Solutions. Langmuir 2016, 32, 11280-11286.

(4) Calgaroto, S.; Wilberg, K. Q.; Rubio, J. On the nanobubbles interfacial properties and future applications in flotation. Miner. Eng. 2014, 60, 33-40.

(5) Zhang, M.; Seddon, J. R. T.; Lemay, S. G. J. Colloid Interface Sci. 2019, 538, 605-610.
(6) Tagit, O.; de Ruiter, M. V.; Brasch, M.; Ma, Y.; Cornelissen, J. J. L. M. Quantum dot encapsulation in virus-like particles with tuneable structural properties and low toxicity. RSC Adv. 2017, 7, 3811038118.

(7) Uchida, M.; Klem, M. T.; Allen, M.; Suci, P.; Flenniken, M.; Gillitzer, E.; Varpness, Z.; Liepold, L. O.; Young, M.; Douglas, T. Biological containers: protein cages as multifunctional nanoplatforms. Adv. Mater. 2007, 19, 1025-1042.

(8) Lee, E. J.; Lee, N. K.; Kim, I.-S. Bioengineered protein-based nanocage for drug delivery. Adv. Drug Delivery Rev. 2016, 106, 157171.

(9) Liu, A.; Traulsen, C. H. H.; Cornelissen, J. J. L. M. Nitroarene reduction by a virus protein cage based nanoreactor. ACS Catal. 2016, 6, 3084-3091.

(10) Brasch, M.; Voets, I. K.; Koay, M. S. T.; Cornelissen, J. J. L. M. Phototriggered cargo release from virus-like assemblies. Faraday Discuss. 2013, 166, 47-57.

(11) Speir, J. A.; Munshi, S.; Wang, G.; Baker, T. S.; Johnson, J. E. Structures of the native and swollen forms of cowpea chlorotic mottle virus determined by $\mathrm{X}$-ray crystallography and cryo-electron microscopy. Structure 1995, 3, 63-78.

(12) Lavelle, L.; Gingery, M.; Phillips, M.; Gelbart, W. M.; Knobler, C. M.; Cadena-Nava, R. D.; Vega-Acosta, J. R.; Pinedo-Torres, L. A.; Ruiz-Garcia, J. Phase Diagram of Self-assembled Viral Capsid Protein Polymorphs. J. Phys. Chem. B 2009, 113, 3813-3819.

(13) van Eldijk, M. B.; Wang, J. C. Y.; Minten, I. J.; Li, C.; Zlotnick, A.; Nolte, R. J. M.; Cornelissen, J. J. L. M.; van Hest, J. C. M. Designing two self-assembly mechanisms into one viral capsid protein. J. Am. Chem. Soc. 2012, 134, 18506-18509.

(14) Caspar, D. L. D.; Klug, A. Physical principles in the construction of regular viruses, Cold Spring Harbor symposia on quantitative biology. Cold Spring Harbor Symp. Quant. Biol. 1962, 27, $1-24$.

(15) Liu, A.; Verwegen, M.; de Ruiter, M. V.; Maassen, S. J.; Traulsen, C. H. H.; Cornelissen, J. J. L. M. Protein cages as containers for gold nanoparticles. J. Phys. Chem. B 2016, 120, 6352-6357.

(16) Verduin, B. J. M. The preparation of CCMV-protein in connection with its association into a spherical particle. FEBS Lett. 1974, 45, 50-54.

(17) Garmann, R. F.; Comas-Garcia, M.; Gopal, A.; Knobler, C. M.; Gelbart, W. M. The assembly pathway of an icosahedral singlestranded RNA virus depends on the strength of inter-subunit attractions. J. Mol. Biol. 2014, 426, 1050-1060.

(18) Vega-Acosta, J. R.; Cadena-Nava, R. D.; Gelbart, W. M.; Knobler, C. M.; Ruiz-García, J. Electrophoretic mobilities of a viral capsid, its capsid protein, and their relation to viral assembly. J. Phys. Chem. B 2014, 118, 1984-1989.

(19) Chou, T.; Jarić, M. V.; Siggia, E. D. Electrostatics of lipid bilayer bending. Biophys. J. 1997, 72, 2042-2055.

(20) Prasad, B. V. V.; Schmid, M. F. Principles of virus structural organization. Adv. Exp Med. Biol. 2012, 726, 17-47.

(21) Verwegen, M. Protein cage clustering: towards functional biohybrid materials; Universiteit Twente, 2014.

(22) Ting, C. L.; Wu, J.; Wang, Z.-G. Thermodynamic basis for the genome to capsid charge relationship in viral encapsidation. Proc. Natl. Acad. Sci. U. S. A. 2011, 108, 16986-16991. 